\title{
Evaluation of the effect of training using auditory stimulation on rhythmic movement in Parkinsonian patients-a combined motor and $\left[{ }^{18}\right.$ F]-FDG PET study
}

\author{
M. Fernández del Olmo, P. Arias, M.C. Furio, M.A. Pozo, J. Cudeiro
}

\begin{abstract}
Introduction: A programme of rehabilitation using auditory cues has previously been shown to decrease movement variability in the gait of Parkinsonian patients.

Objective and methods: We studied the temporal variability of finger-tapping and gait in 9 patients with Parkinson's disease (PD) before and after they undertook a physical rehabilitation programme. Positron Emission Tomography (PET) using 2-deoxy-2 $\left[{ }^{18} \mathrm{~F}\right]$ fluoro-d-glucose (FDG) was performed in these subjects to look for changes in metabolic brain activity after completion of the rehabilitation program.

Results: The reduction of variability was seen not only in gait but also other repetitive movements such as finger tapping. Furthermore, here we show differences in resting regional cerebral glucose utilisation in these patients compared to healthy controls (significant hypometabolism $-p<0.001$-for the PD group in the right parietal and temporal lobes, left temporal and frontal lobes and a hypermetabolism in the left cerebellum) and specific changes following the improvements in repetitive movement abilities (significant metabolic increment $-p<0.001-$ in the PD group in the right cerebellum and in the right parietal and temporal lobes).

Conclusions: Although our study does not allow us to draw firm conclusions, it provides new information on the neural basis of auditory stimulation in PD. Our results extend those from previous studies to show improvement in the temporal variability of two types of rhythmic movements after participation by PD patients in a physical rehabilitation programme, along with changes in glucose uptake in several brain areas involved in sensorimotor processing.
\end{abstract}

Keywords: Rehabilitation; Parkinson; PET; Finger tapping; Gait; rhythm

\section{Introduction}

Parkinson's disease (PD) is clinically characterized by the association of akinesia, rigidity, and resting tremor related to a dopaminergic deficiency of the nigrostriatal pathway. Although patients with PD commonly show slowness and hypometria regarding motor performance, disrupted behaviours that depend upon precise timing, such as rhythmic movement may also be observed. As noted by Stelmach and Worringham [1], timing is a primary aspect of any movement and patients with PD show deficits in the regulation of either force and time parameters, rather than, simply, in force production. Nakamura and colleagues reported that patients had difficulty in maintaining rhythmic movements, such as finger tapping [2], and Hausdorff and colleagues showed that the ability to maintain a steady gait (i.e. low stride-to-stride variability of gait cycle timing and its sub-phases) was diminished in PD (therefore showing high stride-to-stride variability) and the degree of gait variability correlated with disease severity [3]. On the other hand, patients with PD often also exhibit a strong dependence on external cues. There are several examples in support of this: periodic visual inputs from a striped pattern on the floor or a staircase can help PD to sustain locomotion [4], [5] and [6], while in the absence of visual cues patients showed a impairment in the performance of sequential movements [7] and [8]. Furthermore, fingertapping performance was improved in presence of auditory external cues [9].

This dependence on external cues to execute repetitive rhythmic movements has led to a number of intervention strategies in physical therapy designed to improve parkinsonian repetitive movements. Successful examples are rhythmic auditory stimulation (RAS) [10], the metronome therapy [11], and a physical rehabilitation programme (PRP) consisting of a variety of motor tasks performed in combination with rhythmic sound with different cadences [12]. However, research data are yet inconclusive as 
how sensorimotor facilitation can improve brain function and, what is the nature of the underlying mechanisms of recovery.

A number of studies have examined cerebral blood flow changes associated with performance of motor tasks in patients with PD [13], [14] and [15]. These have shown impairments at the levels of the supplementary motor area (SMA) and the dorsolateral prefrontal cortex (DLPFC). However, the lateral premotor system, which is involved in externally triggered movements, seems not to be impaired in PD [14]. While SMA and DLPFC receive a major subcortical input from the basal ganglia, the lateral parietal and premotor areas are targeted by cerebellar projections and are thought to preferentially facilitate instructed rather than freely chosen movements [16]. Interestingly, it has been shown that this circuit is overactivated in PD when patients perform sequential finger movements with one or both hands [17] and [18]. The ability of PD patients to overactivate these lateral cortical areas may explain why they are better able to perform external cued as opposed to freely chosen actions [19].

The purpose of this study was two fold: firstly, following on from a previous study in our laboratory [12], we examined the temporal variability of finger-tapping and gait in patients with PD before and after they undertook our physical rehabilitation programme. Secondly, we used positron emission tomography (PET) using 2-deoxy-2 $\left[{ }^{18} \mathrm{~F}\right]$ fluoro-d-glucose (FDG) in these subjects to look for changes in metabolic brain activity after completion of the Rehabilitation Program.

\section{Patients and methods}

\subsection{Subjects}

A total of 14 subjects were successfully recruited for this study, comprising nine patients with idiopathic PD (five men and four women, aged 61.2 \pm 5.22 ) and five age-matched control subjects (two men and three women, aged 63.1 \pm 4.28 ) with no history of neurological disorders. All subjects provided informed consent according to the Declaration of Helsinki (1964). PD patients were excluded if they had musculoskeletal, cardiovascular or visual disturbances that affected walking ability, or a score $<20$ on the short test of mental status [20], excluding the co-existence of significant dementia. A fundamental requirement for being included in the study was the ability to walk five times up and down a $30 \mathrm{~m}$ walkway without walking aids or assistance. For PD patients the level of functional disability was also determined by the mean of the unified parkinson's disease rating scales (UPDRS) [21], and the Hoehn and Yahr scale [22]. Table 1 shows the specific characteristics of the PD group. All patients showed clinical asymmetry. Four Parkinson's disease patients had an akinetic-rigid phenotype, and five patients had a tremor-dominant phenotype. Drug treatment was kept unchanged throughout the study.

Table 1. Demographic and clinical data of PD patients and control subjects

\begin{tabular}{|c|c|c|c|c|c|c|c|c|}
\hline $1(\mathrm{~F})$ & 64 & 6 & Akinetic & Right & 25 & 2 & 90 & 26 \\
\hline $2(\mathrm{~F})$ & 62 & 6 & Tremor & Right & 25 & 1 & 90 & 23 \\
\hline $3(\mathrm{M})$ & 68 & 8 & Tremor & Left & 31 & 2 & 90 & 33 \\
\hline $4(\mathrm{~F})$ & 65 & 8 & Tremor & Right & 33 & 2.5 & 80 & 45 \\
\hline 7 (M) & 65 & 4 & Akinetic & Right & 27 & 2 & 90 & 27 \\
\hline $8(\mathrm{M})$ & 53 & 4 & Tremor & Right & 33 & 1 & 90 & 12 \\
\hline $9(\mathrm{M})$ & 55 & 3 & Akinetic & Left & 32 & 2 & 90 & 32 \\
\hline Control & & & Age & & & Dominant hand & & \\
\hline $3(\mathrm{~F})$ & & & 66 & & & Right & & \\
\hline $4(\mathrm{~F})$ & & & 63 & & & Right & & \\
\hline $5(\mathrm{~F})$ & & & 55 & & & Right & & \\
\hline
\end{tabular}

Phenotype, akinetic-rigid, or tremor-dominant; laterality, side of body that is most affected by Parkinson's disease; M, male; F, female; STMS, short test of mental status; UPDRS, unified parkinson's disease rating scales. 


\subsection{Procedure}

Firstly, both PD and control groups were evaluated in the basic motor tasks as described below. The following day each subject was evaluated by PET. After this, the PD group began the physical rehabilitation programme. All treatment sessions lasted $1 \mathrm{~h}$ per day, five days a week for 4 weeks. The aim of this programme was to improve (minimize) the temporal variability of finger tapping at preferred diadochokinesis rate (Comfort) and the temporal variability of gait at preferred speed in Parkinson's disease patients. To improve the temporal stability of the finger tapping new exercises were attached to the original physical rehabilitation programme described in the first work [12]. All movements were performed under two different conditions; in the presence of rhythmic auditory cues (synchronized task) or without auditory cues (reproduction task) but patients were never asked to perform the finger-tapping or gait tests (this means at preferred frequency) during the programme (i.e. no direct training in the test tasks). The program included the following exercises:

(1) Gait without upper limbs movements-e.g. patients were asked to walk reproducing or in synchrony with a rhythmic auditory signal. ${ }^{1}$

(2) Gait with sequential movements of the upper limbs-e.g. patients were asked to walk reproducing or in synchrony with a rhythmic auditory signal ${ }^{1}$ while touching themselves on different parts of the body, always in the same order.

(3) Gait with upper limb rhythmic repetitive movements-e.g. patients were asked to walk reproducing or in synchrony with a rhythmic auditory signal ${ }^{1}$ while repetitively apposing thumb to index finger with one hand.

(4) Gait with upper limbs bimanual movement-e.g. patients were asked to walk reproducing or in synchrony with a rhythmic auditory signal ${ }^{1}$ while manipulating an object with two hands.

(5) Gait with upper simultaneous movement-e.g. patients were asked to walk reproducing or in synchrony with a rhythmic auditory signal ${ }^{1}$ while bowling a basketball.

(6) Repetitive single movements with a one arm. Patients were asked to oppose the index finger to the thumb reproducing or in synchrony with a rhythmic auditory signal. ${ }^{2}$

(7) Repetitive sequential movements with a one arm. Patients were asked to oppose the thumb to the other fingers reproducing or in synchrony with a rhythmic auditory signal. ${ }^{2}$

(8) Repetitive bimanual movements (two arms single movement). Patients were asked to tap with both arms reproducing or in synchrony with a rhythmic auditory signal. ${ }^{2}$

(9) Repetitive bimanual movements (two arms sequential movement). Patients were asked to tap the table with each finger in sequence reproducing or in synchrony with a rhythmic auditory signal. ${ }^{2}$

When the physical therapy was completed the PD group was re-evaluated (in both motor tasks and PET). The patients were always examined at the peak dose effect of their antiparkinsonian medication (ON phase). Controls were evaluated once only and did not undertake the rehabilitation programme.

\subsection{Evaluation conditions}

\subsubsection{Gait parameters}

For evaluation, all subjects walked along a $30 \mathrm{~m}$ flat walkway. The instruction to the subject was simply to 'walk to the end of the walkway, at your preferred speed'. This is called preferred gait. To measure the temporal and spatial parameters of the footstep pattern, a set of switches was worn as insoles inside the shoes, sending data in real time (sampling rate $1 \mathrm{~m} \mathrm{~s}$ ) by mean of a small radio transmitter $(10 \times 5 \times 2 \mathrm{~cm})$, to a radio receiver connected to a computer. The system was also connected to two pairs of photoelectric cells, one placed at the beginning and one at the end of the walkway.

The dependent variables measured for each condition of gait included: velocity $(\mathrm{m} / \mathrm{min})$; step length $(\mathrm{m})$; cadence (steps/min) and the coefficient of variation $(\mathrm{CV})$ of recorded intervals between two consecutive steps (in $\mathrm{ms}) . \mathrm{CV}$ is an indicator of temporal variability of gait, where $\mathrm{CV}=(\mathrm{Standard}$ deviation/mean) $\times 100$. 


\subsubsection{Finger tapping test}

All participants performed repetitive flexion-extension movements with their index finger. Subjects were asked to tap at their preferred diadochokinesis rate (Comfort) for a period of $30 \mathrm{~s}$. Tapping was performed with the index finger on the more affected side. If both sides showed similar condition in bilaterally affected patients, they were allowed to use the finger of the dominant hand. A flexible metal loop was fitted snugly to the index finger just proximal to the terminal interphalangeal joint. Each time the metal loop on the index finger struck the contact plate it completed an electrical circuit and generated a brief voltage pulse. Subjects were seated with their forearms resting on a support (elbow angle about $90^{\circ}$ ). To prevent wrist flexion/extension, the wrist was immobilised by a cast. This procedure assured that the sequencing task could be accomplished only by a tapping motion of the index finger.

The dependent variables that were measured for each condition of finger tapping included the tapping frequency in $\mathrm{Hz}$ and the coefficient of variation (CV) of interval of two consecutive taps (in ms) as a indicator of temporal variability of tapping.

\subsection{Statistical analysis}

Results were analysed by paired $t$ test (between PD patients before and after Physical Rehabilitation Programme) and by two sample $t$ test (between PD patients and control group).

\subsection{PET scanning procedure}

FDG-PET scans were performed on high-resolution PET camera (ECAT EXACT 47; Siemens, Erlange, Germany, axial field of view $16.2 \mathrm{~cm}$, in-plane resolution $5 \mathrm{~mm}$ with a half maximum in the centre of the field of view), 28 min after intravenous injection of a mean of 185 MBq of FDG. Prior to the study, a ${ }^{68}$ Germanium $-{ }^{68}$ Gallium transmission scan was performed for 5 min to correct the attenuation. All FDG-PET studies were performed with the subjects eyes closed in a darkened room with minimal noise. Adjustable leather straps were used to minimize movement while ensuring the participant's comfort and laser settings in each scan. The number of scans for patient was two (before and after the physical programme) and one for the control subjects.

\subsubsection{Data analysis}

The images were analysed using statistical parametric mapping (SPM2; Wellcome Department of Cognitive Neurology, London, UK) implemented in Matlab (Mathworks, Inc., Natick, MA, USA). In SPM2 the images were first realigned and co-registered to each other. They underwent spatial normalization, which aligns and warps the individual images into a standard reference space. The data were smoothed using a Gaussian filter $(16-\mathrm{mm})$ to reduce error variance due to image noise and smallscale differences in functional and gyral anatomy. Proportional scaling was used to adjust for differences in global activity between scans. Effects were estimated according to the General Linear Model at each and every voxel. Thus a single average image was produced for each group and images show voxels of significant $Z$ score overlayed onto a standard Talairach MRI-derived brain model, using SPM2. Two types of statistical analysis were performed: comparisons between the PD patient and control groups (two sample $t$-test) and comparisons between the PD patient group scanned pre- and post-Physical Rehabilitation Programme (paired $t$-test). Each comparison gave both significant positive and negative results, each of which are reported here. Results are reported at a threshold of $Z>3.70 \quad(p<0.0001$, uncorrected) for structures without $a$ priori hypothesis. Anatomical locations were identified according to the atlas of Talairach and Tournou [23].

\section{Results}

\subsection{PD group before therapy vs control group}

Table 2 shows the mean values for step length, cadence, velocity, coefficient of variation of gait in the Parkinson's disease group compared with control group. The cadence and coefficient of variation of finger tapping are also shown. The coefficients of variation for gait and finger tapping were significantly higher in the Parkinson group ( $t=3.245 p<0.01$ and $t=2.483 p<0.05$, respectively). From the rest of parameters evaluated there was only a significant difference for velocity of gait, which was less for the PD group $(t=2.904 p<0.05)$. 
Table 2. Comparison between patients before and after the physical rehabilitation program and control subjects

\begin{tabular}{|c|c|c|c|}
\hline \multirow[t]{2}{*}{ Parameter } & \multicolumn{2}{|l|}{$\mathrm{Pd}$} & \multirow[t]{2}{*}{ Control } \\
\hline & Before & After & \\
\hline \multicolumn{4}{|l|}{ Gait } \\
\hline Velocity (m/min) and & $70.79(6.52)$ & $69.97(6.88)$ & $80.60(4.98)$ \\
\hline Cadence (steps/min) & $110.99(7.91)$ & $109.33(10.06)$ & $117.05(2.71)$ \\
\hline \multicolumn{4}{|l|}{ Finger tapping } \\
\hline Frequency $(\mathrm{Hz})$ & $2.55(1.16)$ & $1.81(0.62)$ & $1.67(0.50)$ \\
\hline Coefficient of variation ${ }^{\text {and }}$ & $14.80(12.79)$ & $6,39(3.02)$ & $4,16(0.86)$ \\
\hline
\end{tabular}

Means and standard deviations. The superscript letters show statically significant differences between:

a PD before the physical rehabilitation and control subjects.

b PD after the physical rehabilitation and control subjects.

c PD before and after the physical rehabilitation.

Using PET we detected a significant hypometabolism for the PD group in the right parietal and temporal lobes $(z=4.42 p<0.001)$, left temporal lobe $(z=4.38 p<0.001)$, left frontal lobe $(z=4.00 p<0.001)$ and a hypermetabolism in the left cerebellum (culmen of anterior lobe, $z=3.74 p<0.001$ ) ( Table 3 and Fig. 1).

Table 3. PD group before therapy vs control group

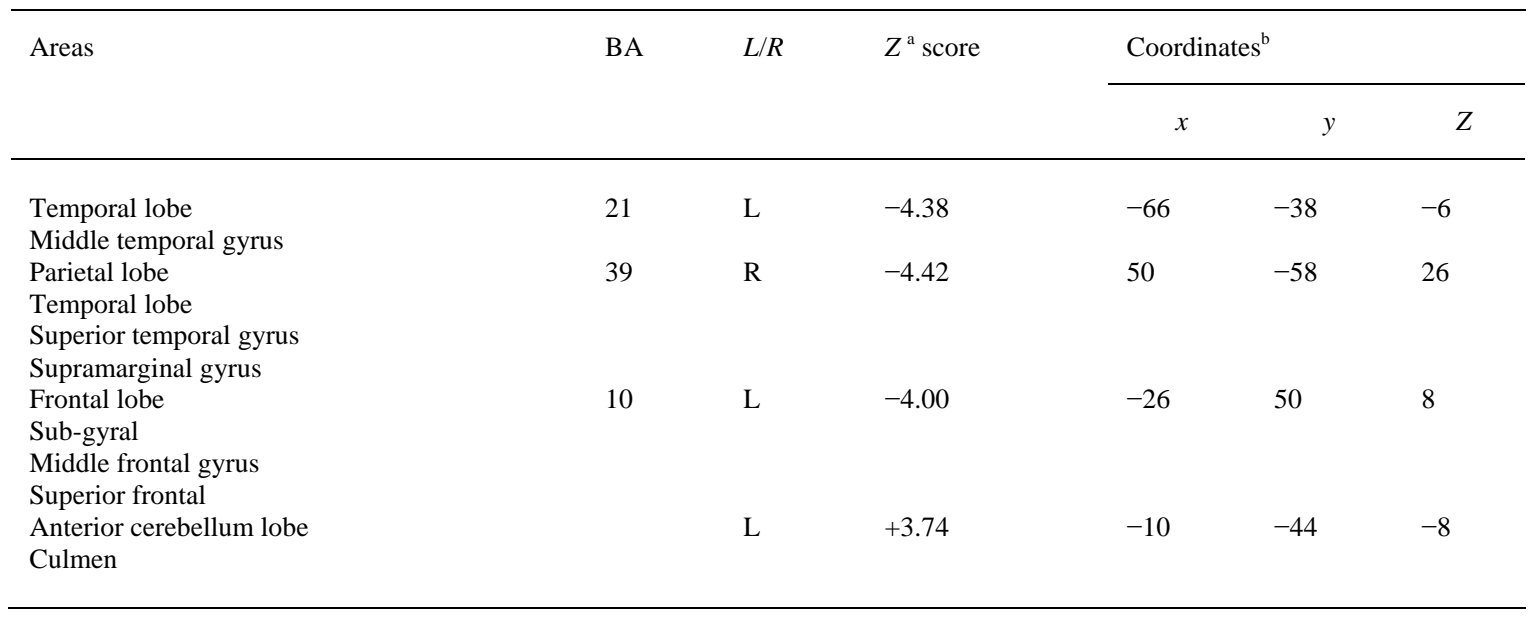

a Table Negative values of the $Z$ score show reductions in glucose metabolism in Parkinson's disease patients compared to normal subjects. Positive values of $Z$ show hypermetabolism in Parkinson's disease patients compared to normal subjects.

b Converted to Talairach space. BA, Brodmann's area; L, left; R, right.

\subsection{PD group before vs PD group after therapy}

Upon completion of the 20 session program, PD subjects demonstrated a statistically significant decrement in the coefficient of variation obtained for finger-tapping and gait $(t=2.107 p<0.05$ and $t=3.887$ $p<0.01$, respectively). The new values of CV recorded for the patients did not statistically differ from those obtained for control subjects ( Fig. 2). However, the rest of the observed parameters did not show any significant change in the PD subjects at the end of the programme ( Table 2). 

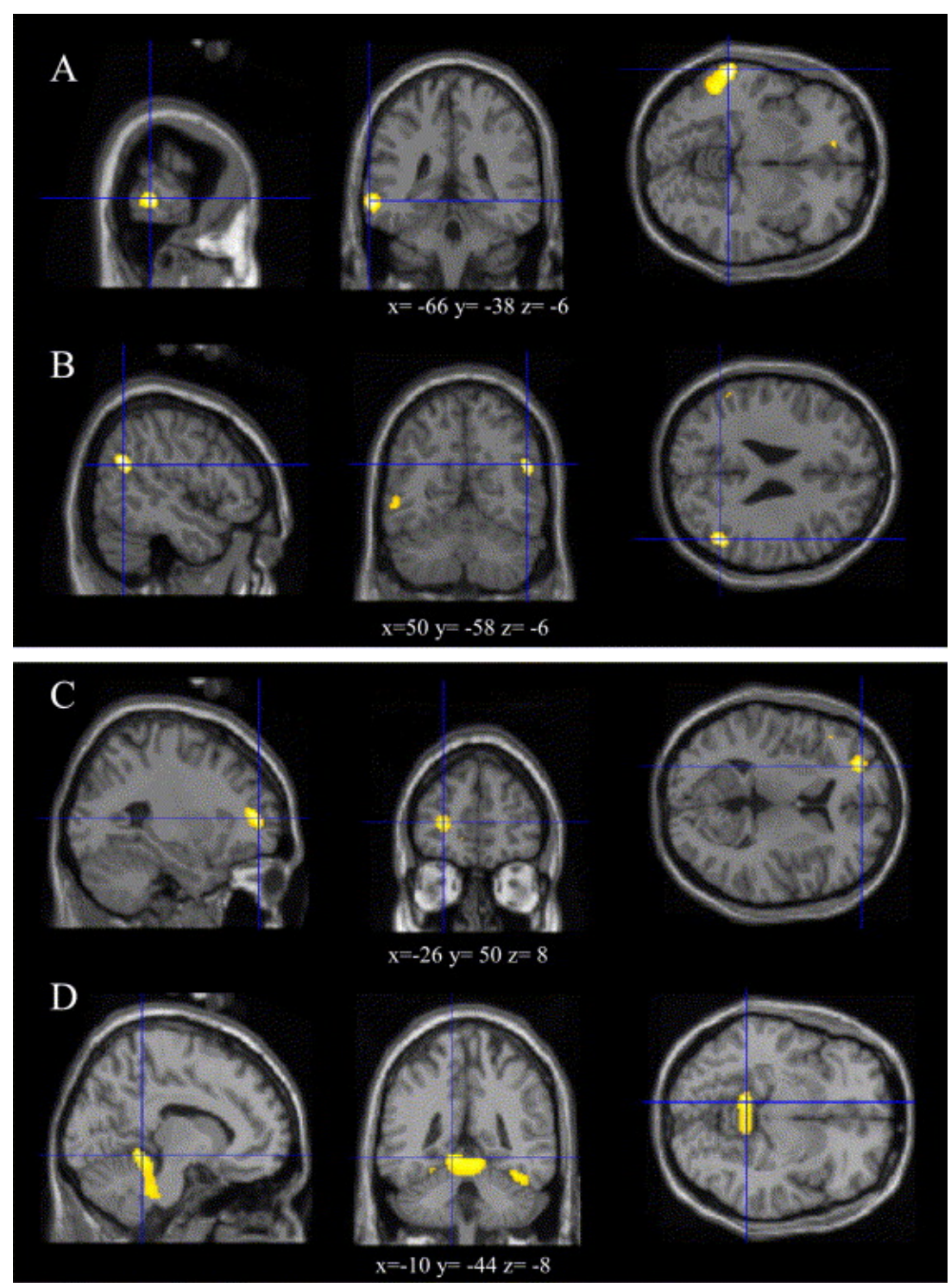

Fig. 1. Location of areas of the brain where there was significant abnormal glucose uptake in Parkinson's disease patients compared to normal subjects. (A)-(C) show a significant hypometabolism in PD patients. (D) show a significant hypermetabolism in PD patients. A: left temporal lobe B: right temporal lobe, right parietal lobe, right superior temporal gyrus; C: left frontal lobe, left subgyral, left middle frontal gyrus, left superior frontal gyrus; D. Left anterior cerebellar lobe, culmen.
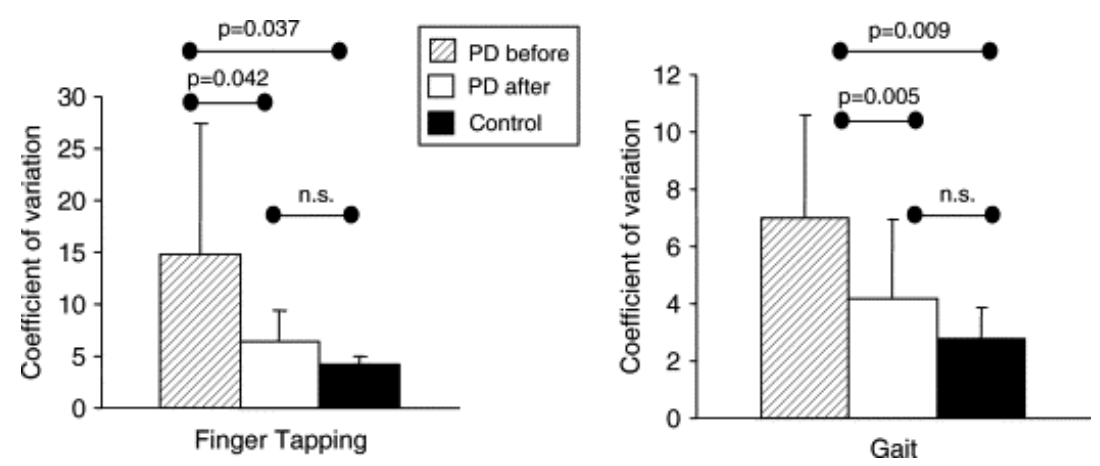

Fig. 2. The coefficient of variation in the PD patients decreased significantly after therapy for finger tapping task (left) and for gait (right). Before therapy the coefficient of variation in PD patients was significantly higher than control subjects. After therapy the coefficient was not significantly different from controls but was significantly different from pretraining levels both for finger tapping and gait. 
Table 4. FDG-PET Data. PD group before vs after therapy

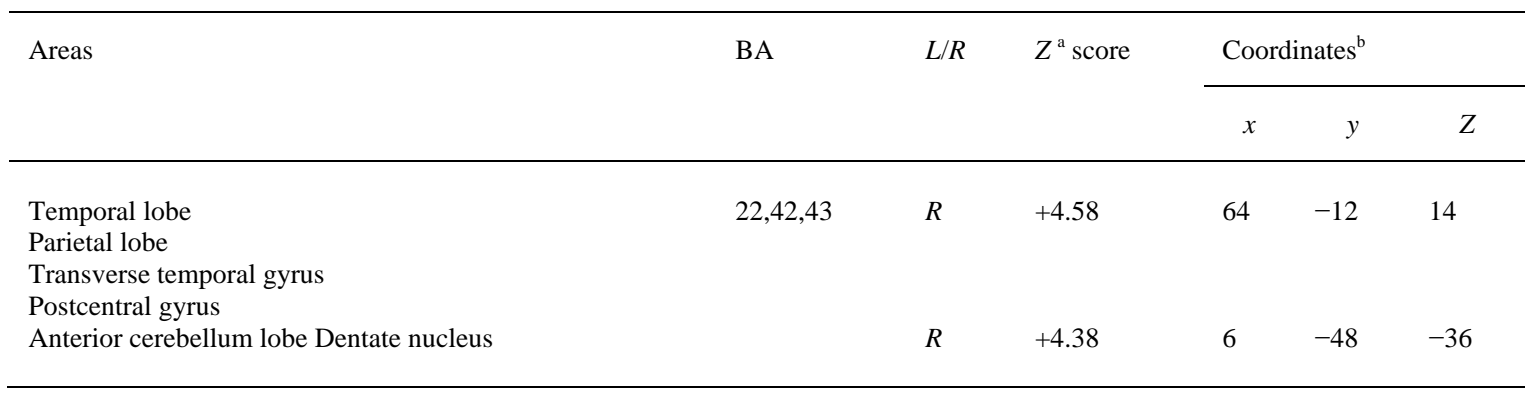

a Table Positive values of $Z$ show increased metabolism in Parkinson's disease patients after physical therapy compared with before physical therapy.

b Converted to Talairach space. BA, Brodmann's area; L, left; R, right.

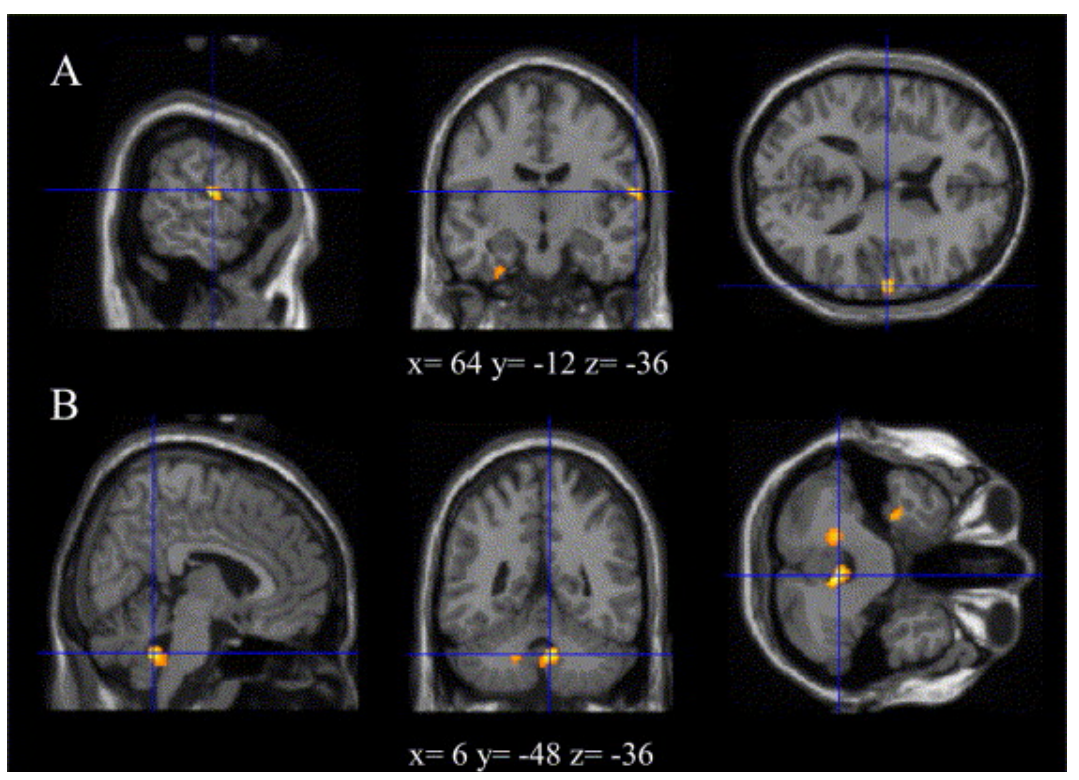

Fig. 3. Location of areas of the brain where there were significant changes in glucose uptake between PD patients before and after therapy. (A) and (B) show a significant increase in PD patients after therapy. A: right parietal lobe, right temporal lobe, right transverse temporal gyrus, right postcentral gyrus; (B): cerebellar lobe, right dentate nucleus.

\section{Discussion}

Following on from our recent study [12], here we present further evidence that our PRP enhances the ability of PD patients to perform repetitive motor tasks, using a regime of training over a 1 month period. The data now not only show this in the case of variability of gait in a walking test, but also for finger tapping. Further, we now demonstrate concomitant changes in cerebral glucose utilisation using FDG in a PET paradigm, comparing activity before and after.

\subsection{PD group before therapy vs control group}

In our experiment, PD patients showed greater variability than control subjects in the motor tasks under study, finger tapping and gait. These results are in agreement with previous work by ourselves and others and confirm the difficulty that patients with Parkinson's disease have to maintain a rhythmic movement. Temporal variability in the finger-tapping test in patients with PD has been previously demonstrated in various studies [2], [24] and [25] including those with patient receiving dopaminergic replacement therapy [26]. More recently the temporal variability in gait was reported by Hausdorff and colleagues [3], and ourselves [12], and the irregular timing of steps in PD suggests a disturbance of the rhythmic locomotor activity generation [27]. Our results show that the patient group had a significantly 
slower velocity compared with the control group, in agreement with previous studies by others [12], [28] and [29], even though the step length and the cadence were not significantly different between patients and control subjects.

When compared to control subjects, the PET analysis showed that PD patients exhibited a significant hypometabolism in the right and left temporal lobes, right parietal lobe and left frontal lobe, and a hypermetabolism in the cerebellum (left side, culmen of anterior lobule). Previous FDG-PET studies in non-demented patients with Parkinson's disease have produced conflicting results. The studies of Eidelberg and collaborators [30] and [31] by the scaled sub-profile model (a method based on principal component analysis of region-of-interest (ROI) data) have led to a characteristic metabolic profile in Parkinson's disease, the so-called 'Parkinson's disease-related profile (PDRP)', which comprises a relative hypermetabolism in the lentiform nucleus, thalamus, and pons, covarying with a relative hypometabolism of the lateral frontal cortex. Furthermore, a positive correlation was reported between this profile and bradykinesia scores, such that the more severe the bradykinesia the more marked the PDRP [31]. However, studies using the ROI method leave substantial areas of the brain unexplored, and have limited reproducibility and objectivity, and often merge physiologically distinct structures into a single value, (e.g. putamen and globus pallidus), potentially obscuring important findings [32]. Furthermore, these studies were performed when patients were in their OFF state, with antiparkinsonian medication at its least effective [32]. Interestingly, Berding and colleagues found significant correlations in advanced patients with PD between increasing UPDRS scores and decreases in FDG uptake in both frontal cortices and the medial temporal lobe on the right side in the ON state, whereas no correlation was found in the OFF state [33]. In another study, a bilateral decrease in glucose uptake was found in parietal and temporal cortices in non-demented patients [34]. Our results, with hypometabolism in the frontal and temporal lobes, are in agreement with the above mentioned studies [33] and [34], and our patients were always evaluated in the ON state. However, we have not calculated possible correlations between glucose uptake and clinical scores in the present study. The small number of patients and both, the absence of remarkable end of doses effects, and on-off fluctuations, would render underpowered this approach.

We also found hypermetabolism in the cerebellum (midline to left, anterior lobe) in our patient group. The meaning of this result is more difficult to explain. The nigrostriatal pathway and the cerebellum appear to be involved in resting tremor [35]. In fact, several PET studies of PD patients with deep brain stimulation in the ventral intermediate nucleus of the thalamus or in the globus pallidus (GPi) have shown increased glucose uptake in the cerebellar vermis in presence of tremor and a decrease in the absence of tremor [36], [37] and [38]. On the other hand, however, it has been reported that glucose uptake of the cerebellar vermis showed a significant negative correlation with the tremor, such that the more severe tremor the lower the vermian metabolism [32]. Hilker and coworkers [39], compared the effect of highfrequency stimulation of the subthalamic nucleus (STN) and a levodopa-challenge on cerebral metabolic activity by FDG-PET in a single patient and found a marked decrease of regional cerebral metabolic rate of glucose in the rostral cerebellar hemispheres in the STN-on-condition (with an important, concomitant decrease of tremor), while the inferior vermis showed an increase of glucose consumption. This discrepancy may be due to different types of afferent cerebellar input. Furthermore, recent views posit that in preventing the resting tremor from spilling over into voluntary movement, the cerebellum may have a compensatory rather than a causal role in PD and the observed tremor-related hyperactivity may be related to the cerebellum actively limiting the rhythmic activity produced by the basal ganglia [37]. It is important to note that, like the physiological studies, these PET studies were carried out during the peak of activity of the antiparkinsonian medication for each patient.

\subsection{PD group before vs PD group after therapy}

In our hands, PD patients improved the regularity of timing in both movements evaluated; this is, finger tapping and gait. Thus, the interval time between taps and between steps became more regular, following therapy (we should emphasise, however, that our study is a pre-post design and we did not include a control patient group which did not receive therapy). This finding agrees with previous studies where walking exercises in the presence of a rhythmic auditory stimulus (RAS) carried out by patients with PD have been shown to be an effective way to improve the temporal pattern of the electric activation in certain muscles, not seen in the absence of the auditory cue [40] and [41]. Although our training paradigm did not improve other parameters like the cadence, velocity or stride length, in other studies patients with PD were able to improve the velocity of their movements after continued practice [42]. This is thought to be an important change since it has been shown that the ability to regulate the stride-to-stride variation in gait timing is impaired in Parkinson's disease patients with freezing of gait compared to those without freezing of gait [43]. In this sense, measurement of gait variability might have a clinical value as a predictive parameter. Furthermore, as suggested by Hausdorff and co-workers the stride-to-stride 
variability in stride time will likely reflect an important aspect of the stride-to-stride control of gait, generally, and stride length, in particular [43].

Finger tapping as a task was chosen as a simple reproducible model for repetitive actions (like gait), which could easily be tested under many different conditions. The work we now report extends our recent observations [12], and suggests that finger tapping might be an alternative task to examine movement variability. The improvement in the temporal variability that we observed in finger tapping after the therapy, supports the idea that walking and finger tapping share many of the same characteristics (being seemingly automatic, periodic) and are regulated by many of the same neural networks (such as basal ganglia, cerebellum) [44] and [45].

With regard to the PET analysis, our results show an increased glucose uptake in the right anterior lobule of the cerebellum and dentate nucleus near the midline and in the right temporo-parietal conjunction after therapy. This increase, together with the improvement in the motor task in our patients after therapy, might mean access to an alternative pathway to compensate the damage to the basal ganglia via SMA to prefrontal circuit, by means of cerebellar projections. While the SMA and DLPFC receive a major subcortical input from the basal ganglia, the lateral parietal and premotor areas are targeted by cerebellar projections. However those projections from cerebellum to cerebral cortex are thought to be mainly contralateral and the activity increase we have shown is ipsilateral. However, dynamic PET studies during the performance of free joystick movements in patients with PD have shown not only a decrease in the activity of supplementary motor area (SMA) and dorsolateral prefrontal cortex (DLPFC), (this is, in the pathway from basal ganglia via SMA to prefrontal cortex), but also increased activity in the cerebellar to parietal to premotor cortex pathway, when this movements was externally triggered [19] and [46]. Thus this pathway could be an adaptive behaviour to dopaminergic deficit and explain why patients with PD improve their movement in presence of external cues [17] and [47]. Although our protocol lacks a control group without training, it is tempting to speculate that this might also include work in this study using external cues in training since it is necessary to remember that our study was a simple examination of resting glucose utilisation, without motor task. We suggest that this activity of the cerebellum, and through it the parietal and premotor areas, would be further increased in the presence of cued movements. Thus the finding that a movement such as finger tapping may improve during our training will permit future works to examine this protocol during dynamic imaging studies.

In the light of our results we cannot to assert that the improvement in timing in our patients after therapy was only due to motor mechanisms but perceptive mechanism too.

Wing and Kristofferson [48] and [49] proposed a two-process model of the analysis of the timing of repetitive movements. In this model two independent processes are assumed to be involved in timing. The first is an internal timekeeper, which triggers the response and so determines when a response should be made, and the second is an implementation system, which executes the motor command. The basal ganglia and cerebellum have been logical candidates for hypothetical timekeeping operations, as damage to these brain regions commonly disrupts behaviours that depend upon precise timing, (such as rhythmic movements in Parkinson's disease [50]) and regulation of agonist-antagonist muscle activity in cerebellar damage [51]. Furthermore, a recent study of used functional MRI provides compelling evidence for the involvement of the basal ganglia in formulating representations of time [52]. Our results did not show an increase in glucose utilisation in the basal ganglia after therapy but did in the dentate nucleus and in the right temporal and parietal lobes. The dentate nucleus is also thought to be involved in timekeeping mechanism [24], [53], [54] and [55] and the inferior right parietal and superior temporal cortex are involved in the coding of temporal intervals [52], [56] and [ 57]. Thus it is possible to suggest that the main adaptation in our patients after therapy would be related to timekeeping more than any motor/effector system. It is important to underline that the all movements performed by the patients during therapy demanded continuous coding of temporal intervals, for the rhythmicity of these movements was always imposed externally by auditory cues under experimental control rather than internally by the patient.

\section{Acknowledgements}

This work was supported by a Xunta de Galicia Grant (PGIDIT02BTF13701PR). We wish to thank Dr Kenneth Grieve for his suggestions and corrections on the manuscript. 


\section{References}

[1] Stelmach GE, Worringham CJ. The preparation and production of isometric force in Parkinson's disease. Neuropsychologia 1988;26:93-104.

[2] Nakamura R, Nagasaki H, Narabayashi H. Disturbances of rhythm formation in patients with Parkinson's disease: Part 1. Characteristics of tapping response to the periodic signals. Percept Mot Skills 1978; 46:63-75.

[3] Hausdorff JM, Cudkowicz ME, Firtion R, Wei JY, Goldberger AL. Gait variability and basal ganglia disorders: stride-to-stride variations of gait cycle timing in Parkinson's disease and Huntington's disease. Mov Disord 1998;13:428-37.

[4] Martin JP. The basal ganglia and posture. London: Pitman Medical; 1967.

[5] Suteerawattananon M, Morris GS, Etnyre BR, Jankovic J, Protas EJ. Effects of visual and auditory cues on gait in individuals with Parkinson's disease. J Neurol Sci 2004;219:63-9.

[6] Morris M, Iansek R, McGinley J, Matyas T, Huxham F. Three-dimensional gait biomechanics in Parkinson’s disease: evidence for a centrally mediated amplitude regulation disorder. Mov Disord 2005; 20:40-50.

[7] Kritikos A, Leahy C, Bradshaw JL, Iansek R, Phillips JG, Bradshaw JA. Contingent and non-contingent auditory cueing in Parkinson's disease. Neuropsychologia 1995;33:1193-203.

[8] Georgiou N, Bradshaw JL, Iansek R, Phillips JG, Mattingley JB, Bradshaw JA. Reduction in external cues and movement sequencing in Parkinson's disease. J Neurol Neurosurg Psychiatry 1994;57:368-70.

[9] Freeman JS, Cody FW, Schady W. The influence of external timing cues upon the rhythmic of voluntary movements in Parkinson's disease. J Neurol Neurosurg Psychiatry 1993;56:1078-84.

[10] McIntosh GC, Brown SH, Rice RR, Thaut MH. Rhythmic auditory-motor facilitation of gait patterns in patients with Parkinson's disease. J Neurol Neurosurg Psychiatry 1997;62:22-6.

[11] Enzensberger W, Oberlander U, Stecker K. Metronome therapy in patients with Parkinson disease. Nervenarzt 1997;68:972-7.

[12] Del Olmo MF, Cudeiro J. Temporal variability of gait in Parkinson disease: effects of a rehabilitation programme based on rhythmic sound cues. Parkinsonism Relat Disord 2005;11:25-33.

[13] Playford ED, Jenkins IH, Passingham RE, Nutt J, Frackowiak RS, Brooks DJ. Impaired mesial frontal and putamen activation in Parkinson's disease: a positron emission tomography study. Ann Neurol 1992;32:151-61.

[14] Jahanshahi M, Jenkins IH, Brown RG, Marsden CD, Passingham RE, Brooks DJ. Self-initiated versus externally triggered movements. I. An investigation using measurement of regional cerebral blood flow with PET and movement-related potentials in normal and Parkinson's disease subjects. Brain 1995;118:913-33.

[15] Brooks DJ. Neuroimagin of movement disorders. In: Watts RL, Koller WC, editors. Movement disorders. Neurologic principles and practice. New York: McGraw-Hill; 1997. p. 31-48.

[16] Thaler DE, Passingham RE. The supplementary motor cortex and internally sirected movement. In: Crossman AR, Sambrook M, editors. Neural mechanism in disorder of movement. London: Libby; 1989. p. 175-81.

[17] Samuel M, Ceballos-Baumann AO, Blin J, Uema T, Boecker H, Passingham RE, Brooks DJ. Evidence for lateral premotor and parietal overactivity in Parkinson's disease during sequential and bimanual movements: a PET study. Brain 1997;120:963-76.

[18] Catalan MJ, Ishii K, Honda M, Samii A, Hallett M. A PET study of sequential finger movements of varying length in patients with Parkinson's disease. Brain 1999;122:483-95.

[19] Brooks DJ. Imaging basal ganglia function. J Anat 2000;196:543-54.

[20] Kokmen E, Naessens JM, Offord KP. A short test of mental status: description and preliminary results. Mayo Clin Proc 1987;62:281-8.

[21] Fahn S, Elton RL. Unifiqued Parkinson's disease rating scale. In: Fahn S, Marsden CD, Calne D, Goldstein M, editors. Recent developments in Parkinson's disease. Florham Park, NY: Macmillan; 1987. p. 153-63.

[22] Hoehn MM, Yahr MD. Parkinsonism: onset, progression, and mortality. Neurology 1967;17:427-42.

[23] Talairach J, Tournoux P. Coplanar stereotaxic atlas of the human brain. Stuttgart: Thieme; 1988.

[24] Ivry RB, Keele SW. Timing functions of the cerebelum. J Cogn Neurosci 1989;1:136-52.

[25] Pastor MA, Artieda J, Jahanshahi M, Obeso JA. Performance of repetitive wrist movement in Parkinson’s disease. Brain 1992;115: 875-91.

[26] O’Boyle DJ, Freeman JS, Cody FWJ. The accuracy and precision of timing of self-paced, repetitive movements in subjects with Parkinson's disease. Brain 1996;119:51-70.

[27] Ebersbach G, Heijmenberg M, Kindermann L, Trottenberg T, Wissel J, Poewe W. Interference of rhythmic constraint on gait in healthy subjects and patients with early Parkinson's disease: evidence for impaired locomotor pattern generation in early Parkinson's disease. Mov Disord 1999;14:619-25.

[28] Morris ME, Iansek R, Matyas TA, Summers JJ. The pathogenesis of gait hypokinesia in Parkinson’s disease. Brain 1994;117:1169-81.

[29] Morris ME, Iansek R, Matyas TA, Summers JJ. Stride length regulation in Parkinson's disease. Normalization strategies and underlying mechanisms. Brain 1996;119:551-68.

[30] Eidelberg D, Moeller JR, Dhawan V, Sidtis JJ, Ginos JZ, Strother SC, Cedarbaum J, Greene P, Fahn S, Rottenberg DA. The metabolic anatomy of Parkinson's disease: complementary [18F] fluorodeox-yglucose and [18] fluorodopa positron emission tomographic studies. Mov Disord 1990;5:203-13.

[31] Eidelberg D, Moeller JR, Dhawan V, Spetsieris P, Takikawa S, Ishikawa T, Chaly T, Robeson W, Margouleff D, Przedborski S. The metabolic topography of parkinsonism. J Cereb Blood Flow Metab 1994;14:783-801.

[32] Lozza C, Marie, RM, Baron JC. The metabolic substrates of bradykinesia and tremor in uncomplicated Parkinson's disease. Neuroimage 2002;17:688-99. 
[33] Berding G, Odin P, Brooks DJ, Nikkhah G, Matthies C, Peschel T, Shing M, Kolbe H, van Den Hoff J, Fricke H, Dengler R, Samii M, Knapp WH. Resting regional cerebral glucose metabolism in advanced Parkinson's disease studied in the off and on conditions with [(18)F]FDG-PET. Mov Disord 2001;16:1014-22.

[34] Hu MT, Taylor-Robinson SD, Chaudhuri KR, Bell JD, Labbe C, Cunningham VJ, Koepp MJ, Hammers A, Morris RG, Turjanski N, Brooks DJ. Cortical dysfunction in non-demented Parkinson's disease patients: a combined (31)P-MRS and (18)FDG-PET study. Brain 2000;123:340-52.

[35] Elble RJ. Diagnostic criteria for essential tremor and differential diagnosis. Neurology 2000;54:2-6.

[36] Parker F, Tzourio N, Blond S, Petit H, Mazoyer B. Evidence for a common network of brain structures involved in parkinsonian tremor and voluntary repetitive movement. Brain Res 1992;584:11-17.

[37] Deiber MP, Pollak P, Passingham R, Landais P, Gervason C, Cinotti L, Friston K, Frackowiak R, Mauguiere F, Benabid AL. Thalamic stimulation and suppression of parkinsonian tremor. Evidence of a cerebellar deactivation using positron emission tomography. Brain 1993;116:267-79.

[38] Davis KD, Taub E, Houle S, Lang AE, Dostrovsky JO, Tasker RR, Lozano AM. Globus pallidus stimulation activates the cortical motor system during alleviation of parkinsonian symptoms. Nat Med 1997; 3:671-4.

[39] Hilker R, Voges J, Thiel A, Ghaemi M, Herholz K, Sturm V, Heiss WD. Deep brain stimulation of the subthalamic nucleus versus levodopa challenge in Parkinson's disease: measuring the on- and off-conditions with FDG-PET. J Neural Transm 2002;109:1257-64.

[40] Thaut MH, McIntosh GC, Rice RR, Miller RA, Rathbun J, Brault JM. Rhythmic auditory stimulation in gait training for Parkinson's disease patients. Mov Disord 1996;11:193-200.

[41] Fernandez del Olmo M, Cudeiro J. A simple procedure using auditory stimuli to improve movement in Parkinson's disease: a pilot study. Neurol Clin Neurophysiol 2003;25:1-7.

[42] Agostino R, Berardelli A, Curra, A, Manfredi M. The performance of rapid arm movements in Parkinson's disease. Adv Neurol 1996;69:135-46.

[43] Hausdorff JM, Schaafsma JD, Balash Y, Bartels AL, Gurevich T, Giladi N. Impaired regulation of stride variability in Parkinson's disease subjects with freezing of gait. Exp Brain Res 2003;149:187-94.

[44] Fukuyama H, Ouchi Y, Matsuzaki S, Nagahama Y, Yamauchi H, Ogawa M, Kimura J, Shibasaki H. Brain functional activity during gait in normal subjects: a SPECT study. Neurosci Lett 1997;228:183-6.

[45] Riecker A, Wildgruber D, Mathiak K, Grodd W, Ackermann H. Parametric analysis of rate-dependent hemodynamic response functions of cortical and subcortical brain structures during auditorily cued finger tapping: a fMRI study. NeuroImage 2003;18:731-9.

[46] Debaere F, Wenderoth N, Sunaert S, Van Hecke P, Swinnen SP. Internal vs external generation of movements: differential neural pathways involved in bimanual coordination performed in the presence or absence of augmented visual feedback. NeuroImage 2003;19:764-76.

[47] Rascol O, Sabatini U, Fabre N, Brefel C, Loubinoux I, Celsis P, Senard JM, Montastruc JL, Chollet F. The ipsilateral cerebellar hemisphere is overactive during hand movements in akinetic parkinsonian patients. Brain 1997;120:103-10.

[48] Wing AM, Kristofferson AB. The timing of interresponse intervals. Percept Psychophys 1973;13:455-60.

[49] Wing AM, Kristofferson AB. Response delays and the timing of discrete motor responses. Percept Psychophys 1973;14:5-12.

[50] Narabayasi H, Nakamura R. Clinical neurophysiology of freezing in parkinsonism of freezing in parkinsonism. In: Delwaide PJ, Agnoli A, editors. Clinical neurophysiology in Parkinsonism. New York; 1985. p. 49-57.

[51] Manto M. Phatophysiology of cerebellar dysmetria: the imbalance between the agonist and the antagonist electromiographic activities. Eur Neurol 1996;36:333-7.

[52] Rao SM, Mayer AR, Harrington DL. The evolution of brain activation during temporal processing. Nat Neurosci 2001;4:317-23.

[53] Casini L, Ivry RB. Effects of divided attention on temporal processing in patients with lesions of the cerebellum or frontal lobe. Neuropsychology 1999;13:10-21.

[54] Ivry RB, Keele SW, Diener HC. Dissociation of the lateral and medial cerebellum in movement timing and movement execution. Exp Brain Res 1988;73:167-80.

[55] Malapani C, Dubois B, Rancurel G, Gibbon J. Cerebellar dysfunctions of temporal processing in the seconds range in humans. NeuroReport 1998;9:3907-12.

[56] Liegeois-Chauvel C, Peretz I, Babai M, Laguitton V, Chauvel P. Contribution of different cortical areas in the temporal lobes to music processing. Brain 1998;121:1853-67.

[57] Platel H, Price C, Baron JC, Wise R, Lambert J, Frackowiak RS, Lechevalier B, Eustache F. The structural components of music perception. A functional anatomical study. Brain 1997;120:229-43. 